\section{Biologische Effekte von Dieselemissionen}

\author{
Schon 1873 war Charles Blackley aufgefallen, dass „Heufieber“ öfter in \\ Städten als auf dem Lande auftritt. Die Hygienehypothese vermochte \\ dies teilweise zu erklären. Doch liegt inzwischen eine Fülle von Daten \\ vor, die einen Zusammenhang zwischen Luftverschmutzung und \\ Herz-Kreislauf- sowie Atemwegserkrankungen aufzeigen.
}

$\mathrm{H}$ auptquelle der Luftverunreinigung sind die Emissionen der mit fossilen Brennstoffen betriebenen Motoren. $\mathrm{Zu}$ unterscheiden sind gasförmige und partikuläre Motorenemissionen, letztere stammen zum Großteil aus Dieselmotoren. Die moderne Dieseltechnologie hat zwar sowohl die Menge an Stickoxiden als auch die Gewichtsmenge an ausgestoßenen Partikeln vermindert, nicht aber deren Zahl. Es überwiegen nunmehr Teilchen kleineren Durchmessers mit relativ großer reaktiver Oberfläche, woraus ein erhöhter biologischer Effekt resultiert.

Zahlreiche Untersuchungen zeigen einen Zusammenhang zwischen Luftverschmutzung und Herz-Kreislauf-Erkrankungen sowie unspezifischen und allergischen Atemwegserkrankungen. Da es an Diesel-spezifischen Biomarkern fehlt, sind ausschließlich Diesel-abhängige Effekte auf die Atemwege schwer zu messen. Dies gilt besonders für Dieselpartikel. Dennoch zeigen epidemiologische sowie sowohl In-vivo- als auch In-vitro-Untersuchungen einen klaren negativen Einfluss von Dieselpartiklen auf die Atemwege.

In verschiedenen epidemiologischen Untersuchungen zeigten Probandenkollektive mit möglichst ausschließlichem Dieselabgas- bzw. Dieselpartikelkontakt vermehrt chronischen Reizhusten, Bronchitis und eine Verschlechterung der Lungenfunktion im Vergleich zur nicht exponierten Kontrolle. Ferner wurde eine erhöhte allergische Sensibilisierungsrate mit Zunahme von Asthma bronchiale eindeutig nachgewiesen, wobei an verkehrsreichen Straßen lebende Kinder besonders gefährdet waren.

In-vivo-Experimente in Inhalationskammern zeigten, dass unter Dieseleinfluss bei den Probanden chronische bronchiale Entzündungen auftraten. Eine Zunahme der bronchialen Hyperreaktivität wurde registriert. Bereits bestehen-

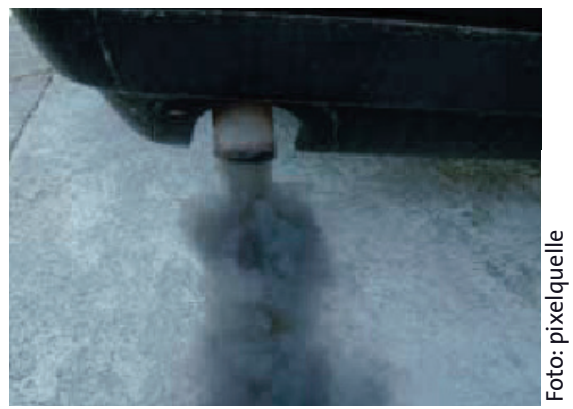

An der Gesundheitsschädigung durch Dieselemissionen kann kein Zweifel mehr sein.

de inhalative Allergien verschlimmerten sich. Bei nasalen Provokationsproben mit einem Gemisch aus Dieselpartikeln und Allergenen zeigte sich eine deutlich gesteigerte allergische Reaktion mit Verstärkung der allergischen Entzündung, eine erhöhte Produktion von Gesamt-IgE und spezifischem IgE sowie eine Vermehrung allergiespezifischer Zytokine.

In-vitro-Untersuchungen mit Dieselpartikeln wurden an unterschiedlichen humanen Zelltypen durchgeführt. Nach Zugabe von Dieselpartikeln sind eine massive Steigerung der IgE-Produktion der B-Zellen, eine Degranulation von
Eosinophilen mit vermehrter Haftfähigkeit an nasalen Epithelzellen und eine Erhöhung der IgE-vermittelten Histaminfreisetzung aus Mastzellen zu beobachten. In verschiedenen Zellen wurde eine vermehrte Bildung von Zytokinen nachgewiesen, welche die Produktion von IgE und der allergischen Entzündung fördern. Im Tierversuch wurden diese Beobachtungen bestätigt.

Die gegenwärtige Forschung versucht die biochemischen Prozesse, welche den Diesel-abhängigen Gewebsveränderungen zugrunde liegen, aufzuklären. Die wichtigste Hypothese geht dabei davon aus, dass die Dieselexposition oxidativen Gewebsstress induziert, indem die körpereigenen, Gewebe reparierenden Prozesse inhibiert werden. Zahlreiche Experimente sprechen für die Richtigkeit dieser Vorstellung.

Forschungsbedarf besteht zum Effekt ultrafeiner, also nicht abfiltrierbarer Dieselpartikel und der Entwicklung klinischer Marker für Diesel-abhängigen oxidativen Gewebsstress. Wichtig wäre auch, die Populationen zu identifizieren, die für Diesel-abhängige Erkrankungen besonders anfällig sind. Schließlich könnten auch Verbesserungen der medikamentösen Therapie möglich sein. Speziell ein therapeutischer Effekt von Antioxidanzien scheint sich hier abzuzeichnen.

\section{Dr. Dieter Bruchhausen, Wuppertal}

\section{Literatur}

Riedl M, Diaz-Sanchez D. Biology of diesel exhaust effects on respiratory function. J Allergy Clin Immunol 2005; 115: 221-8
Freiexemplar für ÄDA-Mitglieder

Unter Beteiligung des ÄDA wurde im Aktionsbündnis Allergieprävention die evidenzbasierte und konsentierte Leitlinie Allergieprävention erarbeitet. Anfang des Jahres ist die Langfassung dieser Leitlinie in Buchform erschienen. Dank der Unterstützung durch das Bundesministerium für Gesundheit und Soziale Sicherung kann auf Anfrage interessierten ÄDAMitgliedern ein Exemplar kostenlos zur Verfügung gestellt werden. Da die Anzahl der ÄDA-Mitglieder größer als die der vorhandenen Bücher ist, muss gegebenenfalls der Zeitpunkt Ihrer Anfrage über die Zuteilung entscheiden.

Ihre Anfrage richten Sie bitte schriftlich an die ÄDA-Geschäftsstelle, Carin Fresle und Ursula Raab Blumenstraße 14, 63303 Dreieich

Fax: (o 6103$) 697019$

E-Mail: raab@sersys.de

Prof. Dr. Torsten Schäfer, Aktionsbündnis Allergieprävention

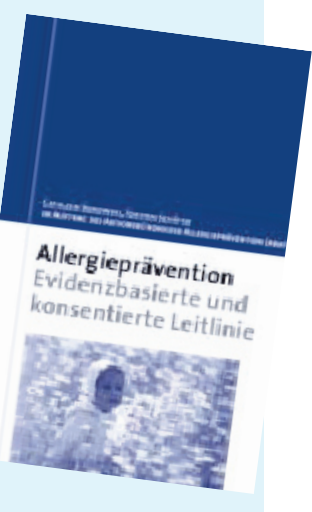

